\title{
Convergence Remedies For Non-Smooth Payoffs in Option Pricing
}

\author{
D. M. Pooley, K. R. Vetzal ${ }^{\dagger}$ and P. A. Forsyth ${ }^{\ddagger}$ \\ University of Waterloo \\ Waterloo, Ontario \\ Canada N2L $3 \mathrm{G} 1^{\S}$
}

June 17, 2002

\begin{abstract}
Discontinuities in the payoff function (or its derivatives) can cause inaccuracies for numerical schemes when pricing financial contracts. In particular, large errors may occur in the estimation of the hedging parameters. Three methods of dealing with discontinuities are discussed in this paper: averaging the initial data, shifting the grid, and a projection method. By themselves, these techniques are not sufficient to restore expected behaviour. However, when combined with a special timestepping method, high accuracy is achieved. Examples are provided for one and two factor option pricing problems.
\end{abstract}

\section{Introduction}

As financial contracts become increasingly complex, so too do pricing methods. In many cases, analytic solutions are infeasible. Effective numerical pricing schemes are therefore required. As discussed in more detail below, option valuation problems commonly feature discontinuities in either the payoff function or its derivatives. This non-smooth data causes quantization error (Tavella and Randall, 2000), and can lead to serious degradation in the convergence rates of numerical schemes. Another consequence can be poor estimates of the solution derivatives (delta and gamma values) even though the prices appear to be correct.

In this article, we provide a simple remedy for these problems. More specifically, we rely on finite element analysis and use a special timestepping method in conjunction with various procedures for smoothing discontinuities. Although the idea of smoothing the payoff has been discussed before in the finance literature (see Heston and Zhou, 2000), this was in the context of vanilla payoffs using explicit type numerical methods which are generally only first order accurate in time. Higher order rates of convergence can sometimes be obtained (Heston and Zhou, 2000), but require special circumstances (i.e. constant volatility). By contrast, we examine cases with discontinuous payoffs, and use Crank-Nicolson type methods which can theoretically attain second order convergence for any volatility structure. We show that this higher order rate can be achieved by employing a combination of some form of smoothing and a modified timestepping method. As a byproduct, we

\footnotetext{
*Department of Computer Science, dmpooley@elora.math.uwaterloo.ca

${ }^{\dagger}$ School of Accountancy, kvetzal@watarts.uwaterloo.ca

${ }^{\ddagger}$ Department of Computer Science, paforsyt@elora.math.uwaterloo.ca

§ Acknowledgement: This work was supported by the Natural Sciences and Engineering Research Council of Canada, the Social Sciences and Humanities Research Council of Canada, and the Royal Bank of Canada.
} 
show how appealing to known partial differential equation (PDE) evaluation and analysis techniques can help to explain and improve upon the observed behaviour of other pricing methods (such as lattices).

Classical convergence results typically rely on smoothness assumptions for the underlying data. However, as noted above, many financial contracts have discontinuities in the payoff conditions or their derivatives. For example, the payoffs of standard vanilla options have discontinuous first derivatives. Contracts where an advance notice of exercise is required (e.g. most callable bonds) also have discontinuous derivative values. Discretely monitored barriers introduce discontinuities at observation dates, while the payoff itself is discontinuous for digital options. To further complicate matters, discontinuities often occur in regions of highest interest, such as near the strike. It is often critical to obtain prices and hedging parameters near these asset values.

The convergence rate of lattice schemes has been analyzed in Heston and Zhou (2000). In that paper it was shown that non-smooth payoff conditions decrease the expected rate of convergence for call options. In this article, we use PDE methods to numerically price digital call options and supershare binary call options. Since lattice methods (i.e. binomial or trinomial trees) can be viewed as explicit finite difference schemes, one would expect similar convergence rate difficulties.

When discretizing in time, many methods are available. Explicit schemes are typically simple to implement, but suffer from stability issues. Implicit methods are unconditionally stable, but only exhibit linear convergence. If possible, it is advantageous to use Crank-Nicolson timestepping to achieve quadratic convergence. However, Crank-Nicolson timestepping is prone to spurious oscillations if twice the maximum stable explicit timestep is exceeded. Further, if discontinuous initial conditions are present, the expected quadratic convergence may not be realized. Another possibility is to use a multi-step method such as a second order backward difference technique. While this approach works well for European options, in our experience multi-step methods do not perform optimally for complex American options (Windcliff et al., 1999). Consequently, it is desirable to use methods which restore second order convergence for Crank-Nicolson timestepping.

Along these lines, Rannacher (1984) proposed a scheme in which Crank-Nicolson timestepping is preceded by a finite number of implicit steps. The rationale is that high frequency error components will be dampened by the implicit steps, leading to smooth convergence. The expected rate of convergence remains quadratic since only a finite number of implicit steps are taken. Furthermore, this type of timestepping can help eliminate oscillations in the solution derivative values. Effective hedging of the underlying contract is then made easier.

Other methods for dealing with initial discontinuities have also been proposed. In Heston and Zhou (2000), an averaging method discussed in Thomée and Wahlbin (1974) was used to improve convergence. In Tavella and Randall (2000), it was suggested that shifting the grid such that discontinuities occur midway between grid points can increase accuracy. Note that this idea is similar to placing nodes equidistantly from a discretely observed barrier (Cheuk and Vorst, 1996). Perhaps the most general method for handling discontinuities was given in Wahlbin (1980). Under this method, the initial conditions undergo an $L_{2}$ projection onto the space spanned by a given set of basis functions.

Below we shall see that both the modification to Crank Nicolson timestepping suggested in Rannacher (1984), and some kind of smoothing method are necessary in order to obtain second order convergence for pricing problems with discontinuous payoffs. Illustrative examples will be provided for both one factor and two factor digital call option problems, and one factor supershare binary call options. 


\section{Background}

For ease of exposition, we will focus on one asset digital call options and one asset supershare binary call options (extensions for multi-factor contracts are briefly discussed in section 4). Note that both of these contracts have discontinuous payoff functions. As our main purpose here is illustrative, we will ignore some technical issues involved in pricing and hedging these contracts (see Chriss and Ong, 1995) and simply assume that the underlying asset price follows geometric Brownian motion and that investors are risk-neutral. Let $t$ be time, $T$ be the expiry date, $V$ be the option value, and $S$ be the underlying asset price. The value of both digital and supershare options satisfies the PDE

$$
\frac{\partial U}{\partial t}+\frac{1}{2} \sigma^{2} S^{2} \frac{\partial^{2} U}{\partial S^{2}}+r S \frac{\partial U}{\partial S}-r U=0,
$$

with appropriate initial and boundary conditions. When pricing backwards in time, the option payoff becomes the initial condition. Classical error estimates for the numerical solution often rely on Taylor series analysis. Such an analysis assumes the existence of all derivatives in the underlying function. In other words, it assumes a certain smoothness in the underlying data. In the cases to be considered, such assumptions are invalid and so the expected rate of convergence may not be achieved in practice.

As previously mentioned, various methods have been proposed to alleviate the difficulties of discontinuous initial conditions. At some level, each method attempts to smooth the initial data without affecting the nature of the problem. Three different methods will be discussed below. In all cases, $f_{i}$ will denote the initial data value at node $i$ on the discrete grid. The function $f(S)=f$ will denote the exact initial conditions, which are assumed to be known at all spatial points. However, we will review our discretization of equation (1) before presenting these methods.

\subsection{Discretization}

Equation (1) can be discretized over the domain $\Omega$ using a standard finite volume approach (Zvan et al., 2001). Let $N_{i}$ be the standard (linear) Lagrange basis functions defined by the $S_{i}$ grid points. Then

$$
\begin{aligned}
N_{i} & =1 \text { at node } i \\
& =0 \text { at all other nodes } \\
\sum_{k} N_{k} & =1 \text { everywhere in the solution domain. }
\end{aligned}
$$

These basis functions, also called hat functions, are shown in Figure 1.

To make the payoff function the initial condition, we price backwards in time by letting $\tau=T-t$. If we let $U_{i}^{n}=U\left(S_{i}, \tau^{n}\right)$ be the value of $U$ at $\left(S_{i}, \tau=n\right)$, then the discretization of equation (1) is given by:

$$
\begin{aligned}
A_{i}\left(\frac{U_{i}^{n+1}-U_{i}^{n}}{\Delta \tau}\right)= & (1-\theta)\left(\sum_{j \in i \pm 1} \gamma_{i j}\left(U_{j}^{n+1}-U_{i}^{n+1}\right)+\sum_{j \in i \pm 1} L_{i j} \cdot r S_{i} \cdot U_{i j+1 / 2}^{n+1}-A_{i} r U_{i}^{n+1}\right) \\
& +\theta\left(\sum_{j \in i \pm 1} \gamma_{i j}\left(U_{j}^{n}-U_{i}^{n}\right)+\sum_{j \in i \pm 1} L_{i j} \cdot r S_{i} \cdot U_{i j+1 / 2}^{n}-A_{i} r U_{i}^{n}\right)
\end{aligned}
$$




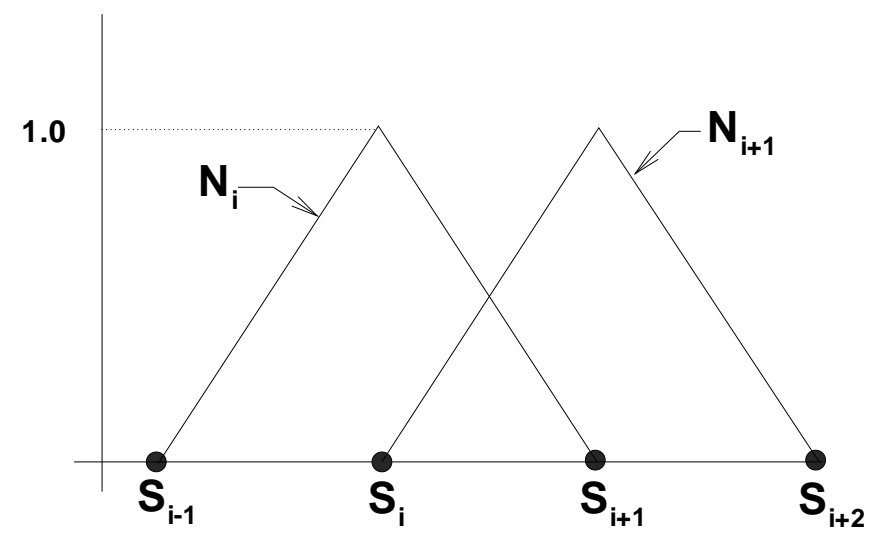

FiguRE 1: Depiction of the standard linear Lagrange basis functions, also called the hat functions.

where:

$$
\begin{aligned}
A_{i}= & \int N_{i} d \Omega=\frac{S_{i+1}-S_{i-1}}{2} \\
\Delta \tau= & \text { timestep } \\
\theta= & \text { timeweighting } \\
& \theta=0 \text { fully implicit } \\
& \theta=1 \text { explicit } \\
& \theta=1 / 2 \text { Crank-Nicolson } \\
\gamma_{i j}= & -\int_{\Omega} \nabla N_{i} \cdot \frac{1}{2} \sigma^{2} S_{i}^{2} \cdot \nabla N_{j} d \Omega=\frac{\sigma^{2} S_{i}^{2}}{2\left|S_{i}-S_{j}\right|} \\
L_{i j}= & \begin{cases}+1 \quad \text { if } j=i+1 \\
-1 \quad \text { if } j=i-1\end{cases} \\
& U_{i}^{n+1}+U_{j}^{n+1} \\
U_{i j+1 / 2}^{n+1}= & 2
\end{aligned}
$$

This discretization may appear overly cumbersome for the simple one factor problems to be studied. However, it can be easily extended to accommodate upstream weighting (if the convection term dominates the diffusion term) and additional underlying factors. For these, and other details, see Zvan et al. (2001).

For the current work, it is important to note that on an equally spaced grid in one space dimension, the standard finite difference, finite element (with mass lumping), and finite volume methods all give identical discretizations. For unequally spaced grids, slight differences may occur, 
especially if upstream weighting (forward/backward differencing) is used for the convection term. Nevertheless, the forms of the discretizations remain the same. In higher dimensions, a finite volume discretization can be considered to be a Galerkin finite element method with a special quadrature rule (Forsyth and Kropinski, 1997). Hence, the finite element results to which we appeal later hold for any standard discretization method in one dimension (equally spaced grid), and are expected to hold for any standard discretization in the more general settings.

\subsection{Averaging The Initial Conditions}

A simple method for handling discontinuities involves averaging the initial data. Specifically, nodal values are replaced with an average value over nearby space. Mathematically, we set $(f(S)$ is the payoff)

$$
f_{i}=\frac{1}{S_{i+1 / 2}-S_{i-1 / 2}} \int_{S_{i-1 / 2}}^{S_{i+1 / 2}} f\left(S_{i}-y\right) d y,
$$

where $S_{i+1 / 2}$ denotes the point halfway between $S_{i}$ and $S_{i+1}$. The validity of this method was proven in Kreiss et al. (1970) and Thomée and Wahlbin (1974). It was used in Heston and Zhou (2000) to increase the rate of convergence when pricing vanilla call options using binomial trees.

\subsection{Shifting The Mesh}

In Tavella and Randall (2000), it was remarked that having the strike price occur midway between mesh nodes generally increases the accuracy of the finite difference method. For an intuitive explanation of this result, consider a digital call option with a strike of 40 dollars, and grid points 39, 40 and 41. In this case, the initial values at these nodes will be 0,1 and 1 respectively. However, the exact same initial values would occur on this grid for any strike price in the range $(39,40]$. In other words, this grid cannot resolve differences between digital options with strike prices just over 39 dollars and up to (and including) 40 dollars. On the other hand, consider having grid points at 39.5 and 40.5. A strike price of 40 then falls halfway between the range of resolvable prices. The maximum possible error is therefore reduced from the previous grid.

On a more mathematical side, note that the averaging procedure defined in Section 2.2 would have no effect on a grid with the strike price halfway between grid points. In a sense, the initial data has already been smoothed. Thus, we might expect this grid to give the expected rate of convergence. However, refining the grid is no longer trivial. Simply adding nodes halfway between existing nodes places a new node at the strike price. To maintain the desired properties of the grid, the refined grid must be shifted such that the strike price once again falls between two grid nodes. Interpolation can be used to recover the option value at the strike price.

\subsection{Projecting The Initial Conditions}

Perhaps the most advanced technique for handling discontinuities involves projecting the initial conditions onto a set of basis functions (Rannacher, 1984). Let $N_{i}$ be the basis functions used in the discretization. In this case, the $N_{i}$ are the linear Lagrange basis functions defined by properties (2). The continuous form of a discrete function can then be written as

$$
c=\sum_{k} c_{k} N_{k}
$$


The best (in the $L_{2}$ norm) approximation of the exact initial conditions $f$ in the space spanned by the $N_{k}$ basis functions is found by selecting $c_{k}$ values to minimize

$$
\int_{\Omega}(c-f)^{2} d \hat{x}
$$

where $\Omega$ is the computational domain. Let $\langle x, y\rangle=\int_{\Omega} x y d \hat{x}$ denote the inner product between functions $x$ and $y$. The solution to problem (6) is also the solution to the linear system $M c=F$ where

$$
M=\left[\begin{array}{cccc}
\left\langle N_{1}, N_{1}\right\rangle & \left\langle N_{1}, N_{2}\right\rangle & \cdots & \left\langle N_{1}, N_{n}\right\rangle \\
\left\langle N_{2}, N_{1}\right\rangle & \left\langle N_{2}, N_{2}\right\rangle & \cdots & \left\langle N_{2}, N_{n}\right\rangle \\
\vdots & \vdots & \ddots & \vdots \\
\left\langle N_{n}, N_{1}\right\rangle & \left\langle N_{n}, N_{2}\right\rangle & \cdots & \left\langle N_{n}, N_{n}\right\rangle
\end{array}\right] \quad \text { and } \quad F=\left[\begin{array}{c}
\left\langle N_{1}, f\right\rangle \\
\left\langle N_{2}, f\right\rangle \\
\vdots \\
\left\langle N_{n}, f\right\rangle
\end{array}\right]
$$

For the simple linear Lagrange basis functions, the entries of $M$ can be computed analytically. The result is

$$
M=\frac{1}{6}\left[\begin{array}{cccccc}
2\left(S_{2}-S_{1}\right) & \left(S_{2}-S_{1}\right) & 0 & 0 & \cdots & 0 \\
\left(S_{2}-S_{1}\right) & 2\left(S_{3}-S_{1}\right) & \left(S_{3}-S_{2}\right) & 0 & \cdots & 0 \\
0 & \left(S_{3}-S_{2}\right) & 2\left(S_{4}-S_{2}\right) & \left(S_{4}-S_{3}\right) & \cdots & 0 \\
\vdots & \vdots & \ddots & \ddots & \ddots & \vdots \\
0 & 0 & \cdots & 0 & \left(S_{n}-S_{n-1}\right) & 2\left(S_{n}-S_{n-1}\right)
\end{array}\right] .
$$

Matrix $M$ is obviously symmetric and diagonally dominant. It is also positive definite and therefore non-singular. Further, by virtue of being tridiagonal, it is relatively quick and simple to solve the resulting linear system.

Entries of $F$ will of course depend on the payoff function $f$. For a known $f$, the entries can be computed analytically. However, it is advantageous to code a black-box routine that can accept any payoff form. If $f$ is piecewise linear, then a second order integration scheme will be exact when using linear basis functions. For example we can use Simpson's rule over each grid interval as follows:

$$
\begin{aligned}
\left\langle N_{i}, f\right\rangle & =\int_{S_{i-1}}^{S_{i}} N_{i} f d x+\int_{S_{i}}^{S_{i+1}} N_{i} f d x \\
& =\frac{S_{i}-S_{i-1}}{2}\left[\frac{4}{6} f\left(S_{i-1 / 2}\right)+\frac{1}{3} f\left(S_{i}\right)\right]+\frac{S_{i+1}-S_{i}}{2}\left[\frac{4}{6} f\left(S_{i+1 / 2}\right)+\frac{1}{3} f\left(S_{i}\right)\right],
\end{aligned}
$$

where $f\left(S_{i-1 / 2}\right)$ is the value of the payoff evaluated halfway between $S_{i}$ and $S_{i-1}$. In practice, for this scheme to be exact for piecewise linear payoff functions, there are two further restrictions:

(i) All discontinuities and/or kinks in the payoff must be aligned with grid nodes.

(ii) To properly handle discontinuities, one must evaluate $f\left(S_{i}-\epsilon\right)$ for the left integral, and $f\left(S_{i}+\epsilon\right)$ for the right integral, instead of $f\left(S_{i}\right)$ in equation (9). Here, $\epsilon$ is some small (positive) value.

From a theoretical point of view, a projection operator $P$ applied to the initial data $f$ has the property that $P^{2} f=P f$. In other words, one application of the projection operator makes the data as smooth as possible in the $L_{2}$ norm. On the other hand, the data resulting from the averaging operator (4) may still have kinks. One may be tempted to average the new data (assuming linear interpolation between the data values), but then it is not clear when this process should end. 


\subsection{Convergence Results From Finite Element Analysis}

It is a commonplace idea that finite difference methods have theoretical difficulties when presented with non-smooth payoffs. In fact, in one dimension the standard discrete finite difference equations are identical to a finite element discretization with linear basis functions and mass-lumping for the time derivative term. Consequently, we can appeal to finite element convergence analysis rather than relying on the classical finite difference analysis which, as noted, assumes smooth data. We can expect that finite element methods will require less continuity than finite difference analysis, since finite element methods are based on the weak or integrated form of the PDE.

The main relevant results (see Rannacher, 1984, for the proof) are as follows. Consider a parabolic PDE, with a non-smooth initial condition (which may contain a finite number of discontinuities). Assuming that the discretization uses linear basis functions and Crank-Nicolson timestepping, second order convergence to the exact solution is obtained for any finite value of $\tau=\tau_{0}>0$, if

i) the initial condition (i.e. the payoff) is $L_{2}$ projected onto the space of basis functions; and

ii) two timesteps of fully implicit timestepping are used, followed by Crank-Nicolson (which we refer to as Rannacher timestepping in the following).

The projection and Rannacher timestepping must occur at all non-smooth states, i.e. whenever a discontinuity is introduced. For digital options, this just means at the start, but in the case of a discretely monitored barrier option, it would be at each monitoring date.

Note that for vanilla payoffs, which are continuous but have discontinuous derivatives, no smoothing is required if there is a node at the strike. This is contrary to the suggestions in Heston and Zhou (2000). In this case, since the payoff can be represented by piecewise linear functions, consistent with the $C^{0}$ basis functions implied by a finite difference method, then the projection operation does not alter the initial data (of course, in the case of a discontinuous payoff, projection does change the initial data).

The above results explain the erratic convergence of the standard binomial lattice method, which is simply an explicit finite difference method on a log spaced grid. When pricing at-themoney options, there is a node at the strike only if the number of timesteps is even. For an odd number of timesteps, the payoff is not in the space of linear basis functions implied by the grid.

\section{Convergence Results}

\subsection{Digital Call Options}

We begin by considering one asset digital call options with payoffs specified by

$$
V(t=T)= \begin{cases}1.0 & \text { if } S \geq K \\ 0.0 & \text { otherwise }\end{cases}
$$

where $K$ is the strike price. The parameters used are listed in Table 1.

Convergence results are presented for a sequence of grids starting with 41 (non-uniform) nodes, and uniformly refined to 641 nodes. Constant timestepping was used, with a step-size of 0.02 for the original grid. The step-size was then cut in half for subsequent refined grids. Note that the ratio of changes in the solution for successive grid refinements will be 2 for a linearly convergent scheme; by contrast with quadratic convergence this ratio will be 4 . 


\begin{tabular}{|l|l|}
\hline Time to expiry & 0.5 years \\
Type & Call \\
$r$ & 0.05 \\
$K$ & 40 \\
$\sigma$ & 0.3 \\
Payoff amount & 1.0 \\
\hline
\end{tabular}

TABLE 1: Model parameters for binary call option.

\begin{tabular}{|c|ccc|ccc|ccc|}
\hline & \multicolumn{3}{|c|}{ Implicit } & \multicolumn{3}{c|}{ Crank-Nicolson } & \multicolumn{3}{c|}{ Rannacher } \\
Nodes & Value & Difference & Ratio & Value & Difference & Ratio & Value & Difference & Ratio \\
\hline 41 & 0.5044549 & & & 0.5027482 & & & 0.5042929 & \\
81 & 0.4981564 & 0.0062985 & & 0.4996981 & 0.0030501 & & 0.4981224 & 0.0061705 & \\
161 & 0.4951500 & 0.0030064 & 2.10 & 0.4967380 & 0.0029601 & 1.03 & 0.4951438 & 0.0029786 & 2.07 \\
321 & 0.4936830 & 0.0014670 & 2.05 & 0.4952843 & 0.0014537 & 2.04 & 0.4936827 & 0.0014612 & 2.04 \\
641 & 0.4929575 & 0.0007255 & 2.02 & 0.4945815 & 0.0007028 & 2.07 & 0.4929580 & 0.0007247 & 2.02 \\
\hline
\end{tabular}

TABLE 2: Convergence results for an at-the-money $(S=40)$ digital call option using various timestepping methods. Data as in Table 1. The exact value is 0.4922403. Difference is the absolute value of the change in the solution as the grid is refined. Ratio is the ratio of successive differences.

To begin, we test the effect of the initial discontinuity on standard timestepping methods. In Table 2, we see that implicit timestepping leads to consistent first order convergence. This is to be expected given the known convergence properties of implicit timestepping. On the other hand, while Crank-Nicolson timestepping has expected quadratic convergence, erratic linear convergence is observed. Using the Rannacher method restores smooth convergence, but only at a linear rate.

In Table 3, we show the results of the three methods for smoothing discontinuities, combined with pure Crank-Nicolson timestepping. All methods appear to have higher convergence, but the rates jump around to such a degree that the convergence is unreliable. In particular, it would be impossible to use the results to extrapolate to a better solution.

Given that the Rannacher timestepping method appears to be beneficial, the tests were repeated with the three smoothing methods combined with Rannacher timestepping. Table 4 shows that all of the methods now give smooth quadratic convergence.

Rannacher timestepping also has a beneficial effect on computed delta and gamma values. During testing, a variety of grids and parameter values showed oscillations in delta and gamma when Crank-Nicolson timestepping was used, even if the payoff was smoothed. For example, Figure 2 plots the gamma of an option using both Rannacher timestepping and Crank-Nicolson timestepping when the initial values are smoothed via the averaging method. This example is based on the same data as in Table 1. We see that Crank-Nicolson timestepping leads to severe oscillations. However, these oscillations disappear when Rannacher timestepping is used.

\begin{tabular}{|c|ccc|ccc|ccc|}
\hline & \multicolumn{3}{|c|}{ Averaging } & \multicolumn{3}{c|}{ Shifting Mesh } & \multicolumn{3}{c|}{ Projection } \\
Nodes & Value & Difference & Ratio & Value & Difference & Ratio & Value & Difference & Ratio \\
\hline 41 & 0.4928843 & & & 0.4926810 & & & & 0.4928886 & \\
81 & 0.4923661 & 0.0005182 & & 0.4923915 & 0.0002895 & & 0.4923299 & 0.0005587 & \\
161 & 0.4922658 & 0.0001003 & 5.17 & 0.4922847 & 0.0001068 & 2.71 & 0.4922505 & 0.0000794 & 7.04 \\
321 & 0.4922437 & 0.0000221 & 4.54 & 0.4922545 & 0.0000302 & 3.53 & 0.4922368 & 0.0000138 & 5.77 \\
641 & 0.4922397 & 0.0000040 & 5.47 & 0.4922454 & 0.0000091 & 3.32 & 0.4922364 & 0.0000004 & 34.4 \\
\hline
\end{tabular}

TABLE 3: Convergence results for an at-the-money $(S=40)$ digital call option using Crank-Nicolson timestepping, combined with various methods for smoothing the payoff. Data as in Table 1. The exact value is 0.4922403. 


\begin{tabular}{|c|c|c|c|c|c|c|c|c|c|}
\hline \multirow[b]{2}{*}{ Nodes } & \multicolumn{3}{|c|}{ Averaging } & \multicolumn{3}{|c|}{ Shifting Mesh } & \multicolumn{3}{|c|}{ Projection } \\
\hline & Value & Difference & Ratio & Value & Difference & Ratio & Value & Difference & Ratio \\
\hline 41 & 0.4928350 & & & 0.4927280 & & & 0.4927897 & & \\
\hline 81 & 0.4923899 & 0.0004451 & & 0.4923676 & 0.0003604 & & 0.4923787 & 0.0004111 & \\
\hline 321 & 0.4922497 & 0.0000281 & 3.99 & 0.4922485 & 0.0000243 & 3.91 & 0.4922490 & 0.0000260 & 3.99 \\
\hline 641 & 0.4922427 & 0.0000070 & 4.00 & 0.4922424 & 0.0000061 & 3.96 & 0.4922425 & 0.0000065 & 4.00 \\
\hline
\end{tabular}

TABLE 4: Convergence results for an at-the-money $(S=40)$ digital call option using Rannacher timestepping, combined with various methods for smoothing the payoff. Data as in Table 1. The exact value is 0.4922403.

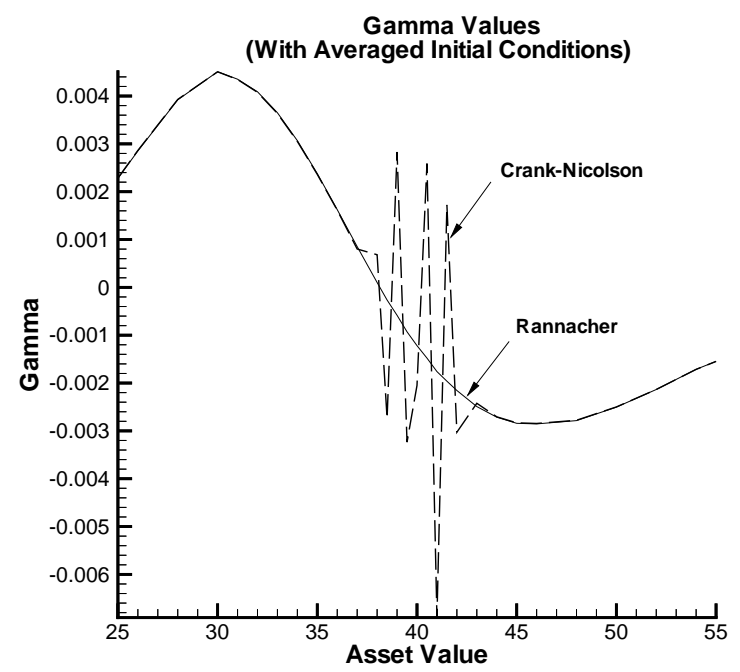

Figure 2: Comparison of gamma values when using Crank-Nicolson timestepping (dashed line) and Rannacher timestepping (solid line) for averaged initial data. Model parameters as in Table 1.

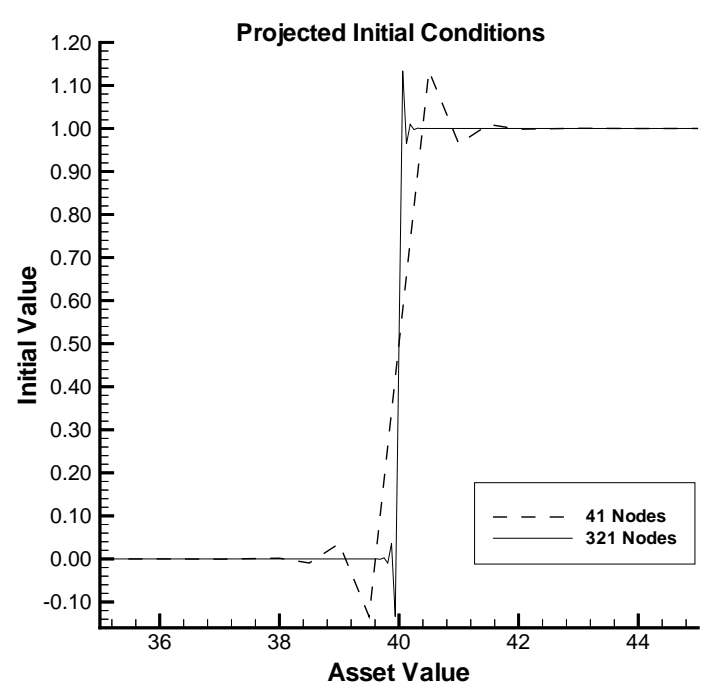

FIGURE 3: Projected initial conditions for the original and fourth refined mesh for a digital payoff.

The effects on the initial data of the averaging method and grid shifting are obvious. The effect of projecting the initial conditions onto the linear basis functions is shown in Figure 3. We see that the projected values overshoot and undershoot the exact values around the strike price. The result is similar to a truncated Fourier series of a step function. Comparing the projected values on the first mesh versus the fourth refined mesh, we see that changes only extend about five nodes beyond the strike price in either direction. The grid spacing and values are not important. While at first sight it is disturbing that the projection values undershoot and overshoot the payoff near the discontinuity, these local extrema are rapidly damped with Rannacher timestepping (as demonstrated by Figure 2). 


\begin{tabular}{|l|l|}
\hline Time to expiry & 1.0 year \\
Type & Call \\
$r$ & 0.05 \\
$K$ & 10 \\
$\sigma$ & 0.2 \\
$d$ & 3 \\
\hline
\end{tabular}

TABLE 5: Model parameters for supershare binary call option.

\begin{tabular}{|c|ccc|ccc|ccc|}
\hline & \multicolumn{3}{|c|}{ Implicit } & \multicolumn{3}{c|}{ Crank-Nicolson } & \multicolumn{3}{c|}{ Rannacher } \\
Nodes & Value & Difference & Ratio & Value & Difference & Ratio & Value & Difference & Ratio \\
\hline 65 & 0.149504 & & & 0.149237 & & & 0.149248 & \\
129 & 0.144083 & 0.005421 & & 0.143952 & 0.005284 & & 0.143955 & 0.005293 \\
257 & 0.141330 & 0.002753 & 1.97 & 0.141266 & 0.002687 & 1.97 & 0.141266 & 0.002689 & 1.97 \\
513 & 0.139943 & 0.001387 & 1.99 & 0.139911 & 0.001355 & 1.98 & 0.139911 & 0.001355 & 1.98 \\
1025 & 0.139248 & 0.000695 & 1.99 & 0.139232 & 0.000679 & 1.99 & 0.139232 & 0.000680 & 1.99 \\
\hline
\end{tabular}

TABLE 6: Convergence results for a supershare binary call option evaluated at $S=K=10$ using various timestepping methods. Data as in Table 5. The exact value is 0.1385509. Difference is the absolute value of the change in the solution as the grid is refined. Ratio is the ratio of successive differences.

\subsection{Supershare Binary Call Options}

To indicate that the results of the previous section are not coincidental, we also test some supershare binary call options. In this case, the payoff is given by

$$
V(t=T)= \begin{cases}0.0 & \text { if } S<K \\ 1.0 / d & \text { if } K \leq S \leq K+d \\ 0.0 & \text { if } S>K+d\end{cases}
$$

where $K$ plays the role of a strike price and $d$ gives the width of the (positive) payoff region. Parameters for the problem are taken from (Dempster et al., 2000) and are summarized in Table 5. Given the two discontinuities (at $K$ and $K+d$ ), we expect this problem to be more difficult than a regular digital call option.

Similar to the digital call option results above, solution values are presented for a sequence of grids starting with 65 (non-uniform) nodes, and uniformly refined to 1025 nodes. The constant stepsize of 0.02 for the original grid was cut in half for each grid refinement.

Results for implicit, Crank-Nicolson and Rannacher timestepping without any data smoothing are given in Table 6. Again, the initial discontinuities have reduced the convergence of both CrankNicolson and Rannacher timestepping to first order. Further, all methods exhibit relatively poor accuracy. Nevertheless, quadratic convergence is once again restored for Rannacher timestepping by smoothing the initial data, as shown by Table 7 . Moreover, accuracy is greatly improved, even on the original grid.

\section{Multi-Factor Extensions}

Each of the three methods for smoothing initial discontinuities can be extended to higher dimensional problems with varying degrees of ease and success. Depending on the payoff condition, it may or may not be possible to place the strike price halfway between nodes in higher dimensions. 


\begin{tabular}{|c|c|c|c|c|c|c|c|c|c|}
\hline \multirow[b]{2}{*}{ Nodes } & \multicolumn{3}{|c|}{ Averaging } & \multicolumn{3}{|c|}{ Shifting Mesh } & \multicolumn{3}{|c|}{ Projection } \\
\hline & Value & Difference & Ratio & Value & Difference & Ratio & Value & Difference & Ratio \\
\hline 65 & 0.1383331 & & & 0.1382546 & & & 0.1384516 & & \\
\hline 129 & 0.1384979 & 0.0001648 & & 0.1384731 & 0.0002185 & & 0.1385275 & 0.0000759 & \\
\hline 257 & 0.1385377 & 0.0000398 & 4.14 & 0.1385310 & 0.0000578 & 3.38 & 0.1385452 & 0.0000177 & 4.30 \\
\hline 513 & 0.1385476 & 0.0000099 & 4.03 & 0.1385459 & 0.0000149 & 3.88 & 0.1385495 & 0.0000043 & 4.10 \\
\hline 1025 & 0.1385501 & 0.0000025 & 4.00 & 0.1385496 & 0.0000038 & 3.93 & 0.1385505 & 0.0000011 & 3.98 \\
\hline
\end{tabular}

TABLE 7: Convergence results for a supershare binary call option evaluated at $S=K=10$ using Rannacher timestepping, combined with various methods for smoothing the payoff. Data as in Table 5. The exact value is 0.1385509 .

\begin{tabular}{|c|ccc|}
\hline Nodes & Value & Difference & Ratio \\
\hline $41 \times 41$ & 0.6893597 & & \\
$81 \times 81$ & 0.6889065 & 0.0004532 & \\
$161 \times 161$ & 0.6887937 & 0.0001128 & 4.02 \\
$321 \times 321$ & 0.6887654 & 0.0000283 & 3.99 \\
$641 \times 641$ & 0.6887583 & 0.0000071 & 4.00 \\
\hline
\end{tabular}

TABLE 8: Convergence results for an at-the-money $\left(S_{1}=S_{2}=40\right)$ two-factor digital call option using Rannacher timestepping and projected initial conditions. The exact value is 0.6887560 .

For example, this smoothing method would be impossible for curved or angled payoff discontinuities when using a structured grid. In theory, both the averaging method and the projection method generalize to higher dimensions. In practice, it may be difficult to accurately integrate the known payoff function if discontinuities are not aligned with element boundaries. This is particularly true for the averaging method, where (technically) the integrations should be done over non-overlapping control volumes surrounding each node.

In any case, we tested the projection method for a simple two-factor digital call option. The pricing equation in this case is given by

$$
\frac{\partial U}{\partial t}+\frac{1}{2} \sigma_{1}^{2} S_{1}^{2} \frac{\partial^{2} U}{\partial S_{1}^{2}}+r S_{1} \frac{\partial U}{\partial S_{1}}+\rho \sigma_{1} \sigma_{2} S_{1} S_{2} \frac{\partial^{2} U}{\partial S_{1} \partial S_{1}}+\frac{1}{2} \sigma_{2}^{2} S_{2}^{2} \frac{\partial^{2} U}{\partial S_{2}^{2}}+r S_{2} \frac{\partial U}{\partial S_{2}}-r U=0 .
$$

The discretization used is given in Zvan et al. (2001), and is essentially an extended form of equation (3). The parameters are the same as in Table 1, with the additions of $\sigma_{1}=\sigma_{2}=0.3$ and an asset correlation of $\rho=0.3$. Further, the payoff condition was taken to be

$$
V\left(S_{1}, S_{2}, t=T\right)= \begin{cases}1.0 & \text { if } \max \left(S_{1}, S_{2}\right) \geq K \\ 0.0 & \text { otherwise. }\end{cases}
$$

The effect of the $L_{2}$ projection is shown on the left side of Figure 4 for the original grid. Subsequent grids were created by splitting each triangle into four new triangles by adding new nodes at all edge midpoints. Despite the rather bumpy nature of the projected initial conditions, Table 8 confirms that the numerical solution obtained by combining the projected initial data with Rannacher timestepping does in fact exhibit quadratic convergence. A plot of the solution surface is shown on the right of Figure 4.

\section{Conclusions}

Discontinuous initial conditions cause problems for standard numerical techniques. In particular, expected convergence rates are not observed and oscillations are more likely to appear in the 

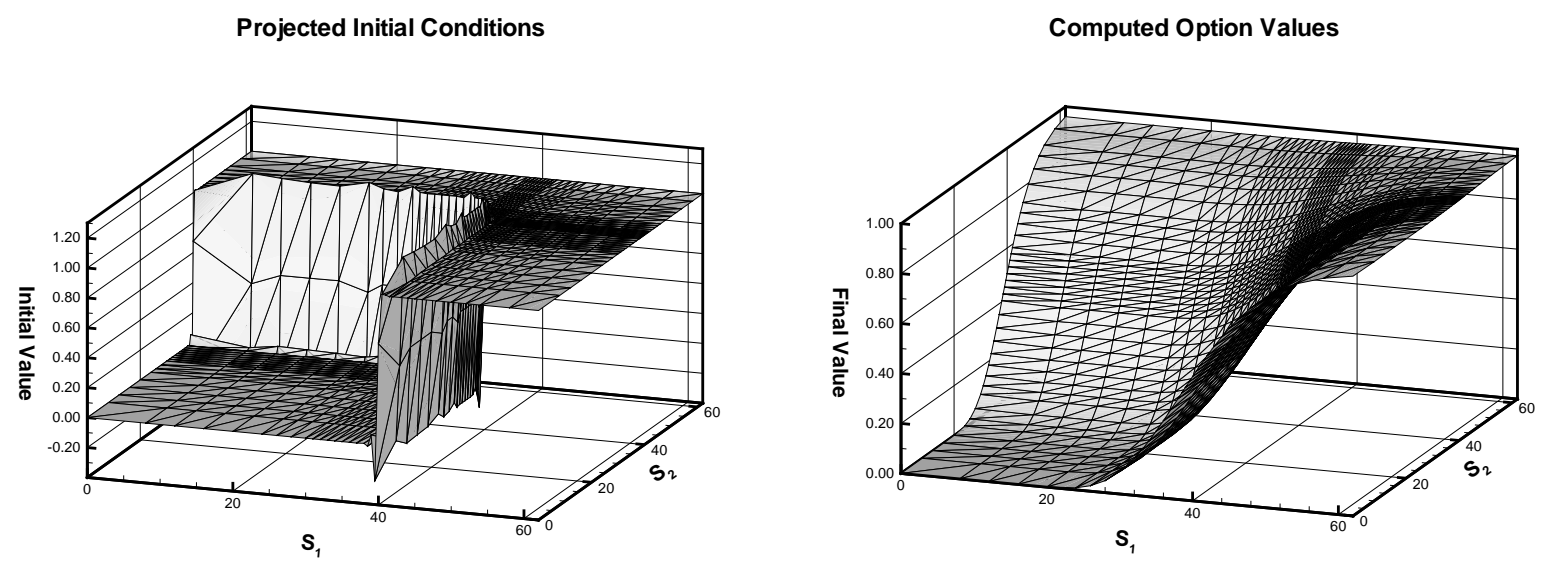

FiguRE 4: Projected initial conditions for a two-dimensional mesh, and the resulting solution.

solution (or its derivatives). In this article, three different techniques for smoothing the payoff were shown to alleviate these problems when used in conjunction with Rannacher timestepping. It is important to note that both smoothing and Rannacher timestepping must be used to obtain second order convergence with discontinuous payoffs.

Rannacher timestepping is simple to implement and greatly improves the stability of the numerical scheme. Its use over Crank-Nicolson timestepping is highly recommended. Of the three schemes for smoothing the payoff, projecting the initial conditions onto the basis function space is perhaps the most difficult to implement. However, it is also arguably the most general. Shifting the grid is simple to do, but may be impossible in higher dimensions, and causes slight problems during grid refinement. Both averaging and projection require numerical integration schemes if they are coded as black-box procedures. There are some technical difficulties with projection in higher dimensions if the discontinuities do not occur at mesh nodes or element faces (Wahlbin, 1980). Studies of these methods in higher dimensions with more complicated payoff discontinuities will be the subject of future research.

\section{References}

Cheuk, T. H. F. and T. C. F. Vorst (1996, Fall). Complex barrier options. Journal of Derivatives 4, $8-22$.

Chriss, N. and M. Ong (1995, December). Digitals defused. Risk 8, 56-59.

Dempster, M., A. Eswaran, D. Richards, and G. Thompson (2000). Wavelet based PDE valuation of derivatives. 7th Annual CAP Workshop on Mathematical Finance, 1 December, 2000.

Forsyth, P. and M. Kropinski (1997). Monotonicity considerations for saturated-unsaturated subsurface flow. SIAM Journal on Scientific Computing 18, 1328-1354.

Heston, S. and G. Zhou (2000). On the rate of convergence of discrete-time contingent claims. Mathematical Finance 10, 53-75. 
Kreiss, H. O., V. Thomée, and O. Widlund (1970). Smoothing of initial data and rates of convergence for parabolic difference equations. Communications on Pure and Applied Mathematics 23, $241-259$.

Rannacher, R. (1984). Finite element solution of diffusion problems with irregular data. Numerische Mathematik 43, 309-327.

Tavella, D. and C. Randall (2000). Pricing Financial Instruments: The Finite Difference Method. Wiley, New York.

Thomée, V. and L. B. Wahlbin (1974). Convergence rates of parabolic difference schemes for non-smooth data. Mathematics of Computation 28(125), 1-13.

Wahlbin, L. B. (1980). A remark on parabolic smoothing and the finite element method. SIAM Journal on Numerical Analysis 17(1), 33-38.

Windcliff, H., P. A. Forsyth, and K. R. Vetzal (1999). Shout options: A framework for pricing contracts which can be modified by the investor. Journal of Computational and Applied Mathematics 134, 213-241.

Zvan, R., P. A. Forsyth, and K. R. Vetzal (2001). A finite volume approach for contingent claims valuation. IMA Journal of Numerical Analysis 21, 703-731. 